\title{
Radiative transfer calculations for a passive microwave satellite sensor: Comparing a fast model and a line-by-line model
}

\author{
S. A. Buehler, ${ }^{1,2}$ N. Courcoux, ${ }^{1}$ and V. O. John ${ }^{1,3}$ \\ Received 3 August 2005; revised 2 May 2006; accepted 23 June 2006; published 21 October 2006.
}

[1] A comparison between the fast radiative transfer model Radiative Transfer for the TIROS Operational Vertical Sounder (RTTOV-7) and the physical radiative transfer model Atmospheric Radiative Transfer Simulator (ARTS) was carried out. Radiances were simulated for the sounding channels of the Advanced Microwave Sounding Unit B (AMSU-B) for the whole globe for a single time of a single day (1 January 2000, 0000 UT). Temperature, pressure, and specific humidity profiles from the reanalysis data set ERA-40 of the European Centre for Medium-Range Weather Forecasts (ECMWF) were used as input for both models; geopotential height profiles were also used but only as input for ARTS. The simulations were made for two different surface emissivities, 0.60 and 0.95 . The low surface emissivity case exhibits the larger radiance differences. Although the global values of the mean difference and standard deviation are small (for example, the global mean difference for channel 18 is $0.014 \mathrm{~K}$ and the standard deviation is $0.232 \mathrm{~K}$ ), the examination of the geographical distribution of the differences shows that large positive or negative values are observed over dry regions of high northern and southern latitudes and over dry elevated regions. The origin of these differences was found to be due to errors introduced by the transmittance parametrization used in RTTOV.

Citation: Buehler, S. A., N. Courcoux, and V. O. John (2006), Radiative transfer calculations for a passive microwave satellite sensor: Comparing a fast model and a line-by-line model, J. Geophys. Res., 111, D20304, doi:10.1029/2005JD006552.

\section{Introduction}

[2] Radiative transfer (RT) models, which simulate observed satellite radiances for a given atmospheric state, are used in most atmospheric remote sensing applications. RT models can be categorized into physical models and fast models. Physical models calculate absorption coefficients from the variables of the atmospheric state using line by line calculations that make them computationally expensive. An example of a physical model is the Atmospheric Radiative Transfer Simulator (ARTS), which is described further in section 2.1. For applications such as the assimilation of satellite data into numerical weather prediction (NWP) models, faster and at the same time accurate RT models are required. Fast RT models usually achieve this goal by parameterizing the absorption stage. RTTOV (Radiative Transfer for the TIROS Operational Vertical Sounder), which is described further in section 2.2, is an example of a fast model where the local optical depth is parameterized using a linear combination of profile-dependent predictors.

\footnotetext{
${ }^{1}$ Institute of Environmental Physics, University of Bremen, Bremen, Germany.

${ }^{2}$ Now at Department of Space Science, Lulea Technical University, Kiruna, Sweden.

${ }^{3}$ Now at Rosentiel School of Marine and Atmospheric Science, University of Miami, Miami, Florida, USA.

Copyright 2006 by the American Geophysical Union. 0148-0227/06/2005JD006552\$09.00
}

[3] The aim of the study presented here was to document systematic and random errors in the fast model RTTOV by using the physical model ARTS as a reference. This was done for the sounding channels of the Advanced Microwave Sounding Unit B (AMSU-B), with most emphasis on channel $18(183.31 \pm 1 \mathrm{GHz})$. The emphasis is on channel 18 because we are planning to use this channel to derive a climatology of upper tropospheric humidity. For this purpose it is crucial that differences between radiances from different RT models are well understood.

[4] Intercomparisons between different RT models have been documented in the literature. They are usually carried out to compare the performances of different models and to document the possible biases. For example, a comparison of different RT models for selected channels of the AMSU and high-resolution infrared radiation sounder (HIRS) instruments is documented by Garand et al. [2001]. (This comparison included RTTOV, but not ARTS, because ARTS was not yet used for AMSU simulations at the time.) The present study is to our knowledge the first where differences between radiances from two different RT models are mapped according to their geographical distribution.

[5] The paper is structured as follows: Section 2 describes the two RT models, the atmospheric input data, and the characteristics of the simulated sensor. It also gives details about the study methodology. Section 3 presents and discusses the simulation results for two different surface emissivity values, representing the two extremes of a low 


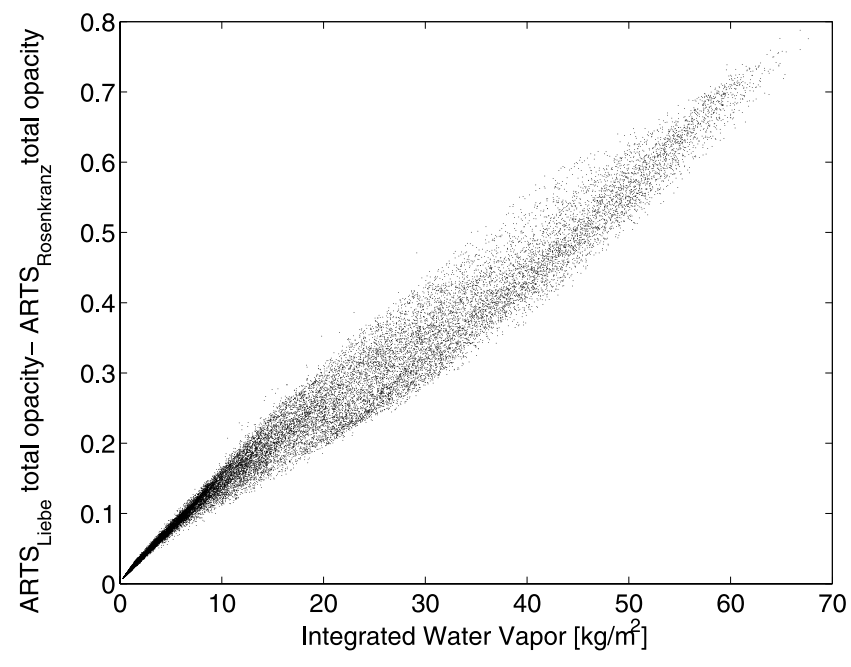

Figure 1. Zenith opacity difference for AMSU channel 18 between absorption combination 1 and 2 (combination 2 minus combination 1) as a function of integrated water vapor (IWV). The actual value of the zenith opacity is approximately 60 at $60 \mathrm{~kg} / \mathrm{m}^{2}$ (compare Figure 2).

emissivity and a high emissivity. Finally, section 4 contains a summary and the conclusions.

\section{Models, Data, and Methodology}

\subsection{ARTS}

[6] The Atmospheric Radiative Transfer Simulator (ARTS) is a physical radiative transfer model that is publicly available. It is described in detail by Buehler et al. [2005]. ARTS is a very general model that can be used from the microwave to the infrared spectral range. Absorption coefficients are obtained by a combination of lineby-line calculation and various continua from the current literature. The model has been compared against a range of other microwave radiative transfer models [Melsheimer et al., 2005] and against collocated AMSU-B data and radiosonde profiles [Buehler et al., 2004]. It has also been used to derive regression coefficients for an upper tropospheric humidity retrieval method from AMSU-B channel 18 data [Buehler and John, 2005]. The arts-1-0-168 version of the model, which was used for the study, simulates only clearsky radiances. There exists another version of ARTS, which includes two different algorithms to simulate the absorption and scattering of radiation by hydrometeors such as clouds and rain [Davis et al., 2005; Emde et al., 2004].

[7] The program setup was as described by Buehler et al. [2004], except that we used two different combinations of absorption models. For the first combination we followed Buehler et al. [2004]: the Rosenkranz [1998] model for water vapor and the Rosenkranz [1993] model for oxygen and nitrogen. For the second combination we followed RTTOV-7: the Liebe [1989] model for water vapor, the Liebe et al. [1992] model for oxygen, and the Liebe et al. [1993] model for nitrogen.

[8] The difference between these two absorption combinations varies with the humidity, as shown by Figure 1. The zenith opacity of the second combination of absorption models is approximately $1 \%$ larger than the zenith opacity of the first combination (compare Figure 2, top).
[9] The weak ozone lines in the AMSU-B frequency range have a considerable impact on the channel 18 brightness temperature [John and Buehler, 2004], but they are not
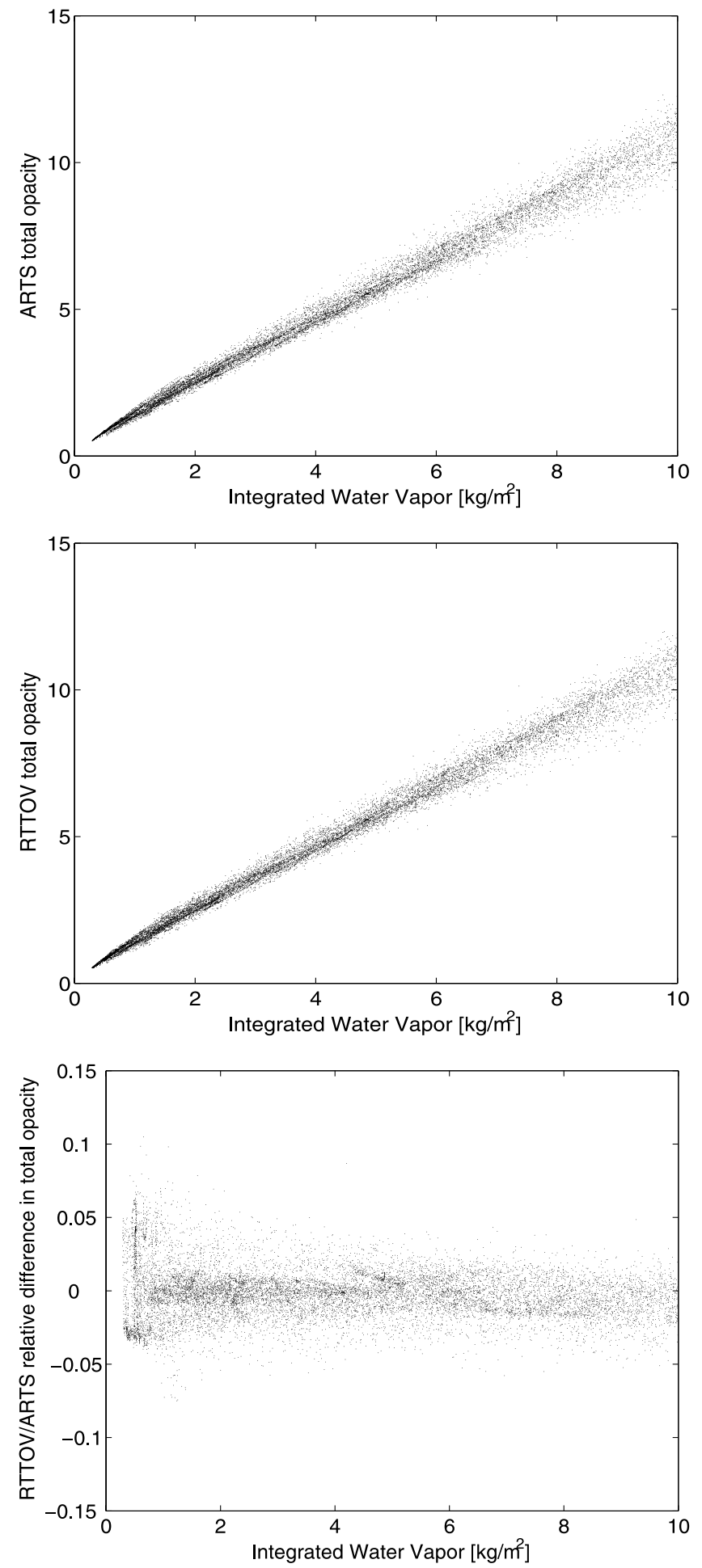

Figure 2. (top) Total opacity calculated by ARTS as a function of IWV, (middle) total opacity calculated by RTTOV as a function of IWV, and (bottom) the relative difference of RTTOV and ARTS total opacities. The calculation used ARTS absorption combination 2. 
included in the simulations as RTTOV also does not include these lines.

[10] As described by Buehler et al. [2004], ARTS takes account of the finite width of instrument passbands, by making explicit RT calculations for a set of frequencies, and then integrating the result.

\subsection{RTTOV}

[11] Radiative Transfer for the TIROS Operational Vertical Sounder (RTTOV) is a fast radiative transfer model which is used by several NWP centers to assimilate satellite radiances into numerical weather prediction models. RTTOV may be also used for other applications that require the simulation of satellite radiances, for example climate monitoring.

[12] RTTOV was originally developed and maintained by the European Centre for Medium-Range Weather Forecasts (ECMWF) but the improvements and updates are now implemented through the EUMETSAT NWP Satellite Application Facility (SAF) by the Met Office, MétéoFrance, and ECMWF, under the coordination of the Met Office. A detailed description of RTTOV is given by Saunders et al. [1999]. The version used for this study is RTTOV-7. By default the atmosphere is divided into 42 homogeneous layers [Saunders, 2002]. In contrast to ARTS, RTTOV does not perform monochromatic RT calculations, but computes a fit to polychromatic transmittances that have been integrated from the Liebe MPM (Millimeter wave Propagation Model) line-by-line model using a diverse dependent set of atmosphere [Rayer, 2001].

[13] At microwave frequencies RTTOV-7 is based on optical depths computed from the Liebe MPM model [Liebe, 1989] with the dry air absorption based upon Liebe et al. [1993], but adjusted so that the dry air calculation effectively comes from Liebe et al. [1992; Rayer, 2001]. RTTOV also includes surface emissivity models for both infrared and microwave channels but they are not used in this study. In addition to the forward model calculation RTTOV can also perform gradient computations which are required for assimilation and retrieval codes.

\subsection{Atmospheric Data}

[14] The atmospheric data used in this study are the ERA-40 reanalysis data produced by the European Centre for Medium-Range Weather Forecasts (ECMWF) [Uppala et al., 2006]. The ERA-40 model uses a T159 spherical harmonic representation corresponding to a maximum horizontal grid spacing of $125 \mathrm{~km}$ and there are 60 levels in the vertical. A date was chosen arbitrarily, 1 January 2000, 0000 UT. Global ERA-40 profiles of temperature, specific humidity, pressure, and geopotential height for this date and time were used.

\subsection{Simulated Radiances}

[15] In this study we simulated AMSU-B radiances. AMSU-B, the Advanced Microwave Sounding Unit B, is flying onboard the satellites NOAA (National Oceanic and Atmospheric Agency) 15, 16, and 17. Details of the instrument can be found in Saunders et al. [1995]. AMSU-B has five channels of which two channels (channels 16 and 17) are surface channels and three channels (18, 19, and 20) are sounding channels that are sensitive to humidity variations at different altitudes with channel 18 the highest peaking one. For tropical or particularly wet midlatitude profiles the sounding altitudes are roughly 8.5, 6.5 and $5 \mathrm{~km}$ for channel 18, 19 and 20 respectively, but for dry condition such as subarctic winter or very dry midlatitude profiles, the sounding altitudes go down to 5.5 and $4 \mathrm{~km}$ for channel 18 and 19 respectively and channel 20 tends to behave more like a surface channel. See Buehler et al. [2004] and Buehler and John [2005] for figures with AMSU-B channel positions and Jacobians.

[16] AMSU is a cross-track scanning instrument, the satellite viewing angle is $\pm 0.55^{\circ}$ from nadir for the two innermost scan positions and $\pm 48.95^{\circ}$ from nadir for the two outermost scan positions. For this comparison the simulations were mainly done for nadir, however the influence of the viewing angle has been studied by doing one calculation for an extreme off nadir viewing angle.

\subsection{Methodology}

[17] The ERA-40 reanalysis fields were interpolated onto a $1.5^{\circ}$ horizontal grid. The comparison was consequently done for 29040 profiles corresponding to the 240 longitudes and 121 latitudes on which data were available. The number of profiles combined with the fact that these profiles cover the whole surface of both Earth hemispheres mean that statistically these profiles roughly represent the natural variability of temperature and specific humidity. For each profile, temperature, specific humidity, and geopotential height from the ERA-40 reanalysis were interpolated onto those RTTOV pressure levels which were located above the surface. Furthermore, the surface pressure was set to the lowest RTTOV pressure level above the true surface. This was done in order to avoid that the surface is located between two RTTOV pressure levels, a case that is handled differently by the two RT models, and that would therefore lead to differences.

[18] Radiances for the AMSU-B sounding channels were simulated by ARTS and RTTOV using the interpolated profiles as input, that means that the radiative transfer equation was integrated over the same vertical levels by both models. For both models the surface emissivity was kept constant and initially equal to 0.60 . In a second step, the simulation was repeated with a surface emissivity of 0.95 . These two emissivity values were chosen because ground emissivity ranges from about 0.60 for ocean to about 0.95 for land. Results are expressed in terms of radiance differences (in units of equivalent black body brightness temperature (BT)) between RTTOV and ARTS, with $\Delta \mathrm{BT}=\mathrm{BT}_{\mathrm{RTTOV}}-\mathrm{BT}_{\mathrm{ARTS}}$.

\section{Results and Discussion}

[19] In this section we show and discuss the results of the simulations. We discuss the low surface emissivity $(0.60)$ case in detail and briefly summarize the results of the high surface emissivity $(0.95)$ case. The possible sources of the observed radiance differences are also discussed.

[20] Figure 3 (top) shows the $\Delta \mathrm{BT}$ for channel 18 on a geographical map (left) and as a function of integrated water vapor (IWV, right). In this case the ARTS calculations were performed using standard ARTS absorption model (combination 1). While the overall agreement is good, two types of 

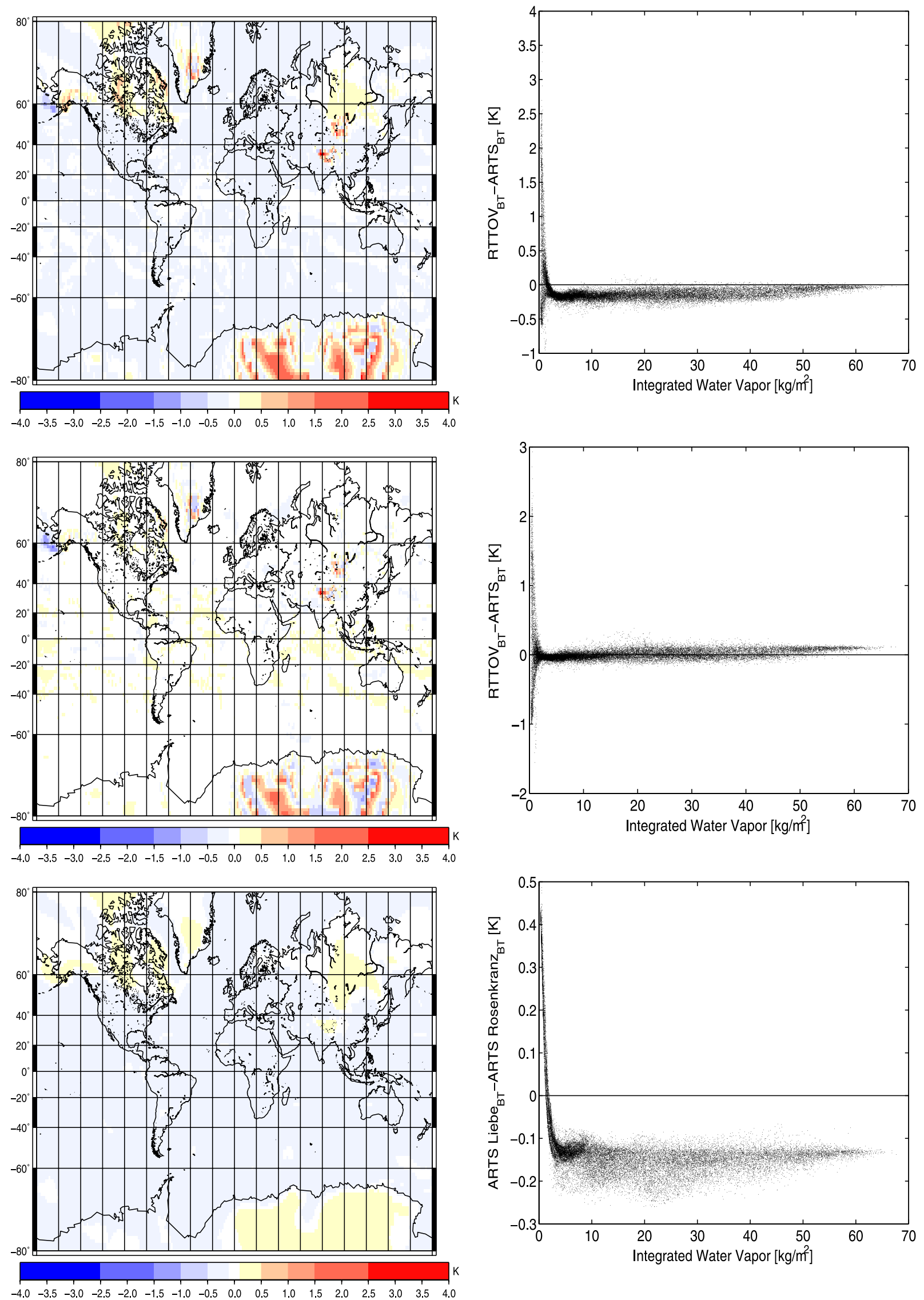

Figure 3 


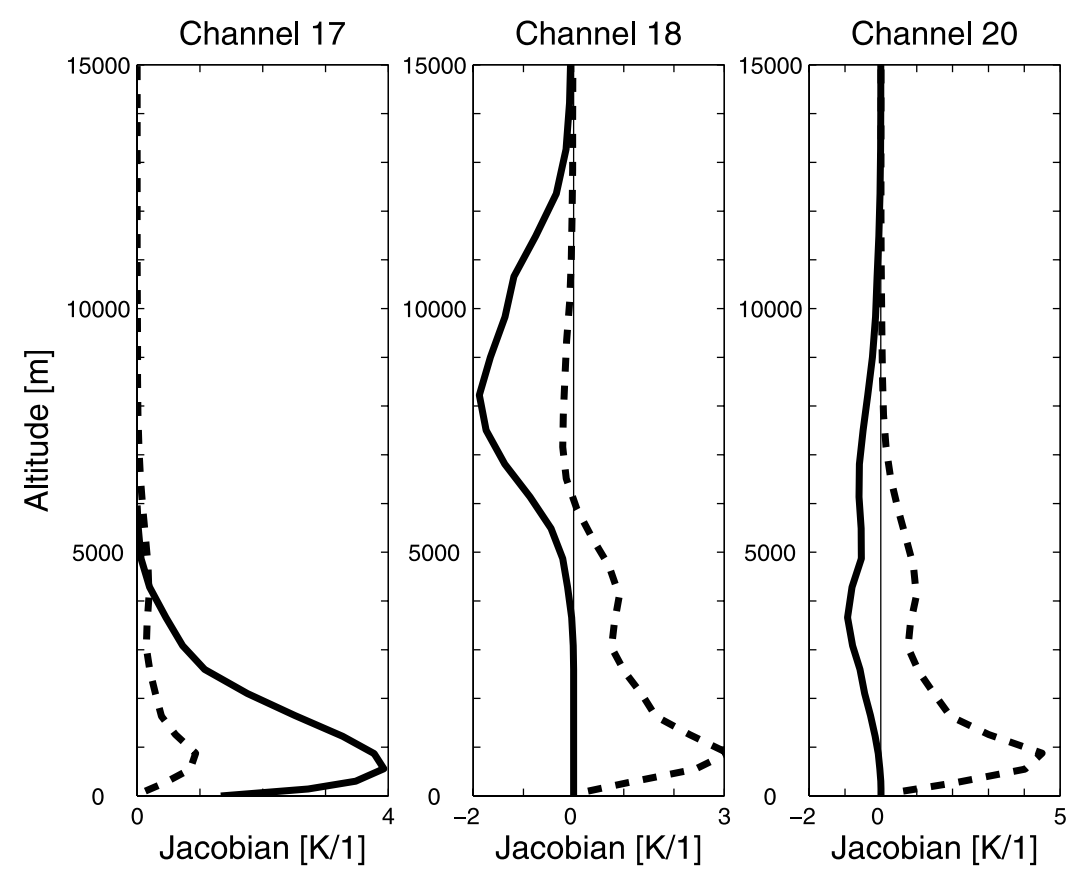

Figure 4. AMSU-B humidity Jacobians in fractional units computed by ARTS. The fractional units are such that the values correspond to the change in brightness temperature for a doubling of the mixing ratio at each level in turn. Jacobians for three different AMSU channels are shown: (left) channel 17, (middle) channel 18, and (right) channel 20. For each channel, two different Jacobians are plotted, one for a normally moist profile (IWV $=32.8 \mathrm{~kg} / \mathrm{m}^{2}$ ) denoted by the solid line and one for a very dry profile $\left(\mathrm{IWV}=1 \mathrm{~kg} / \mathrm{m}^{2}\right)$ denoted by the dashed line.

differences between the models can be seen. First, there is a well spread small negative bias of less than $0.5 \mathrm{~K}$, and secondly, there are larger differences in specific regions, which could be significant for climate oriented studies, as well as for NWP applications. The $\triangle \mathrm{BT}$ becomes large for small IWV values, below approximately $2.5 \mathrm{~kg} / \mathrm{m}^{2}$. Positive $\Delta \mathrm{BT}$ values reach $3.5 \mathrm{~K}$ and negative values reach $-1 \mathrm{~K}$. This result is different from the one obtained by Garand et al. [2001] for a comparison of an older version of RTTOV (version 6) against a range of physical models, since they reported only positive differences for low IWV cases.

[21] Because the differences seen in Figure 3 (top) may be thought to be due to the use of different absorption models, Figure 3 (middle) shows the same results, except that the ARTS calculation used the same absorption models (combination 2) as RTTOV. This results in an overall better agreement. The well spread small negative bias disappears. The agreement is also better for moderate IWV values, but differences are now larger for very high IWV values, and large differences are still visible for very dry profiles. Not all dry profiles exhibit a large radiance difference. For channel 18, approximately $48 \%$ of the profiles with an IWV below $2.5 \mathrm{~kg} / \mathrm{m}^{2}$ have a $\Delta \mathrm{BT}$ between -0.1 and $0.1 \mathrm{~K}$.
[22] In Figure 3 (bottom) we show the difference between ARTS calculations using the two different absorption model combinations. It can be seen that the higher opacity of the absorption combination 2 results in larger BTs for the very dry profiles and smaller BTs for the rest of the profiles. This behavior can be understood with the help of Figure 4, which shows AMSU-B Jacobians (the derivative of BT with respect to humidity changes) for moist and dry profiles. For channel 18 the humidity Jacobian is negative for normally moist profiles, but positive for very dry profiles. Accordingly, an increase in water vapor absorption will decrease BTs for normally moist profiles, but increase BTs for very dry profiles. We can conclude that the differences between the two absorption model combinations, although not being negligible (between $-0.2 \mathrm{~K}$ and $0.5 \mathrm{~K}$ ) do not account for the greatest BT differences between both models. In what follows we will discuss results only for the absorption model combination 2 .

[23] Coming back to the comparison of RTTOV against ARTS, the results shown so far mean that only a small part of the observed differences can be explained by the small differences in the underlying absorption model. Thus, in order to further investigate the sources of the differences one has to look more closely at the opacity values calculated

Figure 3. Radiance differences in Kelvin for channel 18 for simulations with a surface emissivity of 0.60 , using ECMWF analysis profiles of 1 January 2000, 0000 UT as input. (left) Differences displayed on a map; (right) differences displayed as a function of the amount of integrated water vapor (IWV). (top) Difference between RTTOV and ARTS for ARTS absorption model combination 1, the setting of Buehler et al. [2004]. (middle) Same as Figure 3 (top) for ARTS absorption model combination 2, selected to match RTTOV as closely as possible. (bottom) Difference between the two ARTS absorption model combinations (ARTS absorption models combination 2 minus ARTS absorption models combination 1). 

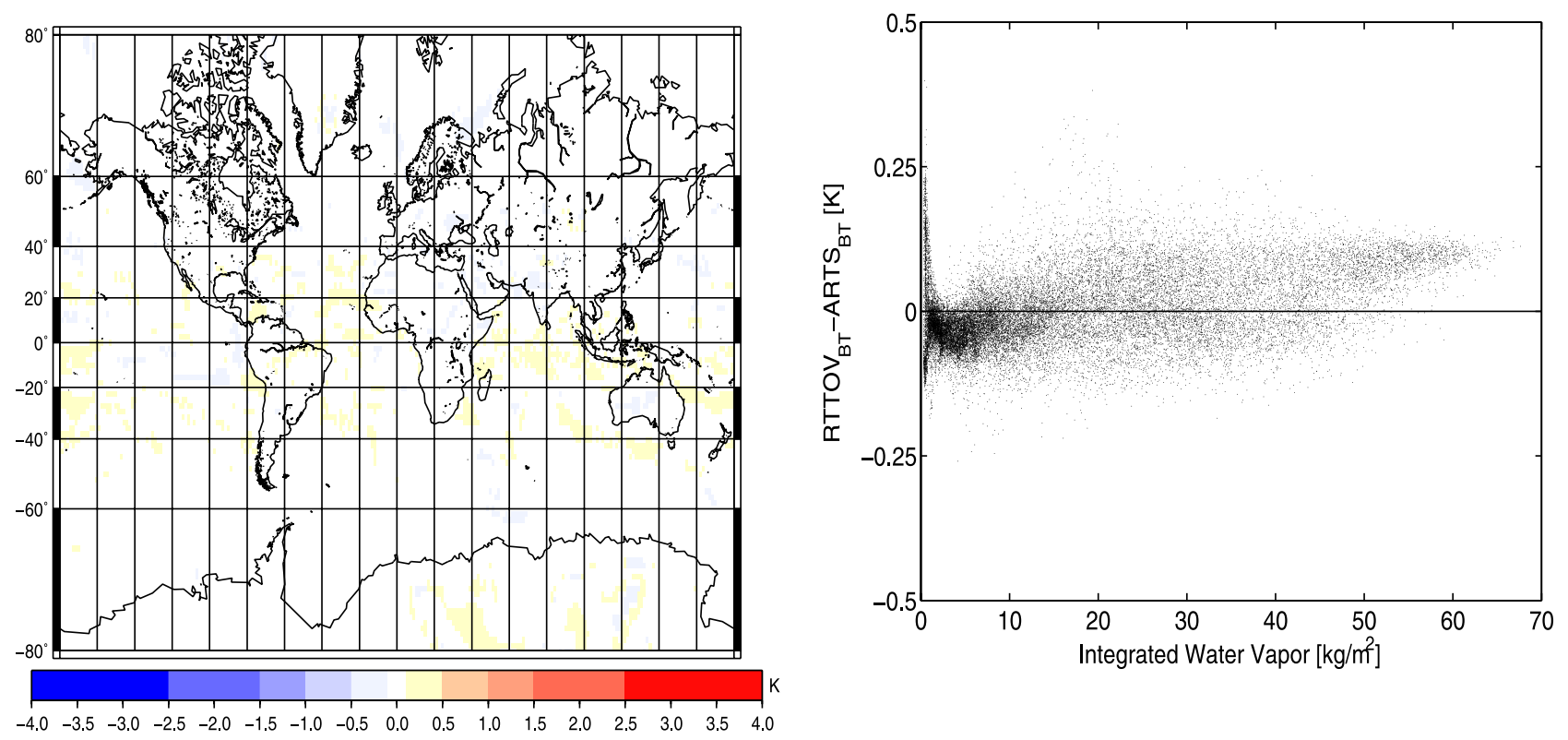

Figure 5. Same as Figure 3 (middle) except that the emissivity is 0.95 . The calculation used ARTS absorption combination 2 .

by RTTOV and ARTS. (In fact, RTTOV outputs the layerto-space transmittance, but this can be easily transformed to the opacity.)

[24] Figure 2 shows the total zenith opacity as a function of IWV for both models, and their relative difference. Figure 2 focuses on the IWV range up to $10 \mathrm{~kg} / \mathrm{m}^{2}$, because for higher water vapor content the transmittance to space gets so low that it cannot be expected to be correctly represented by RTTOV. (In the full data set IWV reaches up to approximately $70 \mathrm{~kg} / \mathrm{m}^{2}$, with a corresponding opacity of about 70.) We expect the opacity to be a linear function of IWV to a good approximation. Both models show this expected behavior.

[25] Figure 2 (bottom) shows the relative difference in total zenith opacity between the two models. It seems to be mostly random. The value exceeds $\pm 10 \%$ in some cases, so it is up to an order of magnitude larger than the difference between the two considered absorption combinations. This indicates that the main difference between RTTOV and ARTS is noise in the RTTOV approximation of the layerto-space transmittance.

[26] In RTTOV the local optical depths are parameterized using a linear combination of profile-dependent predictors. The prediction could be bad for profile values outside the valid range of the regression. However, it was verified that all profiles, even the very dry ones, are within the RTTOV profile validity limits. The RTTOV validity limits were carefully chosen to insure that the prediction is valid. They are currently set to $10 \%$ below and above the minimum and maximum profile values used in the regression, which was verified to be safe by the RTTOV developers (R. Saunders, personal communication). Nevertheless, we observe a certain limitation of RTTOV for cold and extremely dry profiles that are close to the RTTOV validity limit. For such cases RTTOV accuracy is reduced relative to ARTS.

[27] It should be noted that large differences in opacity (or in absorption) are not necessarily translated into large brightness temperature differences. However, there can be large BT differences in cases where the Jacobian peaks near the surface, which happens in elevated and/or extremely dry areas. In that case the BT differences can be amplified by the surface reflection, because any difference in atmospheric modeling is seen twice: once in downward emission, once in upward emission. This effect should depend on the surface emissivity, because the contribution of the reflected downwelling radiation to the upwelling radiation is more important for a low surface emissivity than for a high surface emissivity. (Note that both models were configured to use a simple specular reflection surface model for the comparison.)

[28] To investigate the influence of the surface emissivity, a separate set of calculations with a higher emissivity of 0.95 was performed. Figure 5 shows these simulations for channel 18. As usual, the left panel shows the radiance differences on a map and the right panel shows $\Delta \mathrm{BT}$ versus IWV. In this case the differences for the extremely dry profiles are much less than in the low emissivity case. The positive differences are up to $0.4 \mathrm{~K}$ and the negative differences are down to $-0.26 \mathrm{~K}$.

[29] The discussion so far has focused on channel 18 , but the results for channels 19 and 20, the other two sounding channels, are similar, as shown by Figure 6 . However, the differences for these channels, for a surface emissivity of 0.6 occur already at higher IWV values. Differences occur below approximately $6.5 \mathrm{~kg} / \mathrm{m}^{2}$ for channel 19 and below approximately $15 \mathrm{~kg} / \mathrm{m}^{2}$ for channel 20 . The lower the sounding altitude of the channel, the more profiles show differences. For channel 18, 19 and 20, the percentage of profiles showing a $\triangle \mathrm{BT}$ outside $\pm 0.1 \mathrm{~K}$ is $17.77 \%, 30.76 \%$, and $52.02 \%$, respectively.

[30] All the results shown correspond to nadir viewing geometry. However, to investigate the influence of the viewing angle a calculation has been done for an extreme off-nadir viewing angle of $48.5^{\circ}$ (corresponding to an 

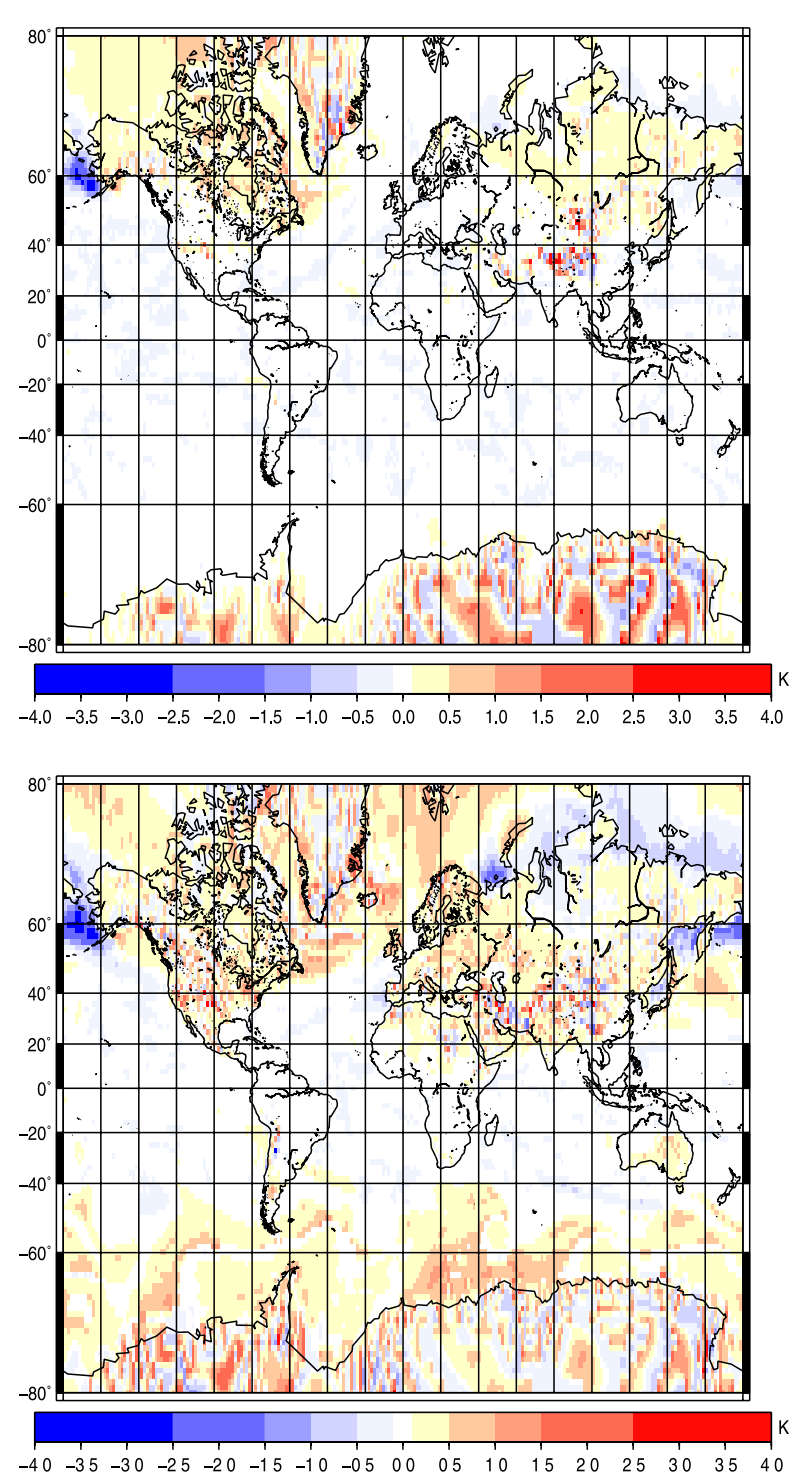
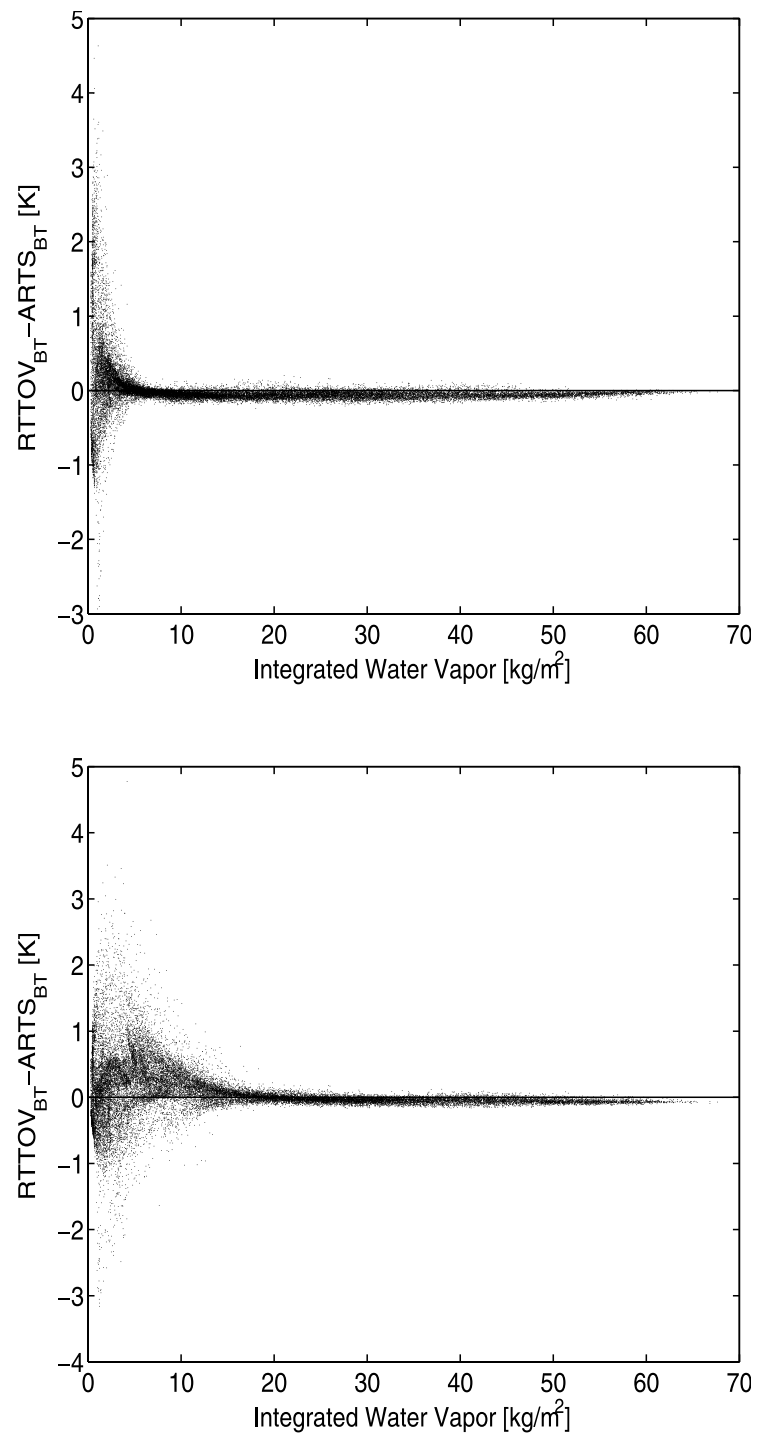

Figure 6. Radiance differences, in Kelvin, for channels 19 and 20, for simulations with a surface emissivity of 0.60 , (left) projected onto a map and (right) as a function of IWV. The calculation used ARTS absorption combination 2.

incidence angle of $58.09^{\circ}$ ). This simulation (not shown) leads to the conclusion that the BT differences between the two models are largest for the nadir view and decrease with increasing viewing angle. The explanation for this behavior is that in the case of an off-nadir viewing angle the opacity of the atmosphere is larger and the Jacobians for the sounding channels peak at higher altitudes. Extreme cases with low opacity and strong surface influence are thus more rare for off-nadir views.

\section{Summary and Conclusions}

[31] A comparison between the fast radiative transfer model RTTOV-7 (Radiative Transfer for TOVS) and the physical radiative transfer model ARTS (Atmospheric Radiative Transfer System) was carried out. The aim of the exercise was to use the physical model ARTS as a reference to test the performance of the fast model RTTOV. Radiances were simulated for the AMSU-B sounding channels by both models for the whole globe for a single time of a single day (1 January 2000, 0000 UT). Temperature, pressure, and specific humidity profiles from the ERA-40 reanalysis were used as inputs for both models, geopotential height profiles were also used but only as input for ARTS. The simulations were made for two different surface emissivities, 0.60 and 0.95 .

[32] Table 1 summarizes the global differences between RTTOV-7 and ARTS absorption model combination 2. The low surface emissivity case has larger differences than the high surface emissivity case. For both emissivity cases, channel 18 has the smallest differences. For the low emissivity case, mean differences range from $0.014 \mathrm{~K}$ for channel 18 to $0.104 \mathrm{~K}$ for channel 20 and the standard deviations range from $0.232 \mathrm{~K}$ for channel 18 to $0.426 \mathrm{~K}$ for channel 20.

[33] A projection onto a map reveals that the greatest differences occur over specific regions (but not all the profiles located in these regions show a difference). Partic- 
Table 1. Summary of Differences Observed Between RTTOV-7 and ARTS $^{\mathrm{a}}$

\begin{tabular}{ccccc}
\hline Channel & $\begin{array}{c}\text { Mean } \\
\text { Difference }\end{array}$ & SD & $\begin{array}{c}\text { Minimum } \\
\text { Difference }\end{array}$ & $\begin{array}{c}\text { Maximum } \\
\text { Difference }\end{array}$ \\
\hline $\begin{array}{c}\text { Low emissivity } \\
18\end{array}$ & & & & \\
19 & 0.014 & 0.232 & -1.55 & 3.07 \\
20 & 0.027 & 0.388 & -2.94 & 4.63 \\
High emissivity & 0.104 & 0.426 & -3.16 & 4.78 \\
18 & & & & \\
19 & -0.002 & 0.063 & -0.26 & 0.40 \\
20 & -0.040 & 0.063 & -0.38 & 0.57 \\
\hline
\end{tabular}

${ }^{\mathrm{a}}$ The different columns show the mean radiance difference $\left(\mathrm{BT}_{\mathrm{RTTOV}}-\right.$ $\mathrm{BT}_{\mathrm{ARTS}}$ ), its standard deviation, the minimum of the difference, and the maximum of the difference. All units are in Kelvin. Results are given separately for the low surface emissivity case $(0.60)$ and the high surface emissivity case $(0.95)$. The calculation used ARTS absorption combination 2.

ularly large positive and negative differences occur over the dry regions of high northern and southern latitude and over dry elevated regions. The differences were found to be mainly due to the errors introduced by the transmittance parametrization used in RTTOV. In particular, it appears that the accuracy of RTTOV is reduced relative to ARTS for profiles having water vapor content close to the lower RTTOV validity limits.

[34] For channel 18, the upper tropospheric humidity channel that is of particular interest to us because we plan to use it for climatological studies, the positive difference in dry regions can exceed $1 \mathrm{~K}$. According to Buehler and John [2005], for channel 18 a $1 \mathrm{~K}$ positive bias in the RT model corresponds to a $7 \%$ relative dry bias in the upper tropospheric humidity. The observed differences may thus be large enough to introduce small regional and seasonal biases in numerical weather prediction model analyses and satellite climatologies, if they are not taken into account.

[35] Acknowledgments. Thanks to ECMWF for providing the ERA-40 reanalysis data. Thanks to Julian Meyer-Arnek and Sandip Dhomse for obtaining and converting the ERA-40 data. Special thanks to Stephen English and Roger Saunders from the Met Office, UK, for discussions on the paper. Thanks also to three anonymous reviewers for their detailed and constructive comments. Thanks to the ARTS radiative transfer community, many of whom have indirectly contributed by implementing features to the ARTS model. RTTOV is developed and maintained by the NWP SAF supported by EUMETSAT, Met Office, ECMWF, and Météo-France. This study was funded by the German Federal Ministry of Education and Research (BMBF), within the AFO2000 project UTH-MOS, grant 07ATC04. It is a contribution to COST Action 723 "Data Exploitation and Modeling for the Upper Troposphere and Lower Stratosphere."

\section{References}

Buehler, S. A., and V. O. John (2005), A simple method to relate microwave radiances to upper tropospheric humidity, J. Geophys. Res., 110, D02110, doi:10.1029/2004JD005111.
Buehler, S. A., M. Kuvatov, V. O. John, U. Leiterer, and H. Dier (2004), Comparison of microwave satellite humidity data and radiosonde profiles: A case study, J. Geophys. Res., 109, D13103, doi:10.1029/ 2004JD004605.

Buehler, S. A., P. Eriksson, T. Kuhn, A. von Engeln, and C. Verdes (2005), ARTS, the atmospheric radiative transfer simulator, J. Quant. Spectrosc. Radiat. Transfer, 91(1), 65-93, doi:10.1016/j.jqsrt.2004.05.051.

Davis, C., C. Emde, and R. Harwood (2005), A 3D polarized reversed monte carlo radiative transfer model for $\mathrm{mm}$ and sub-mm passive remote sensing in cloudy atmospheres, IEEE Trans. Geosci. Remote, 43(6), $1096-1101$

Emde, C., S. A. Buehler, C. Davis, P. Eriksson, T. R. Sreerekha, and C. Teichmann (2004), A polarized discrete ordinate scattering model for simulations of limb and nadir longwave measurements in $1 \mathrm{D} / 3 \mathrm{D}$ spherical atmospheres, J. Geophys. Res., 109, D24207, doi:10.1029/ 2004JD005140.

Garand, L., et al. (2001), Radiance and jacobian intercomparison of radiative transfer models applied to HIRS and AMSU channels, J. Geophys. Res., 106(D20), 24,017-24,031.

John, V. O., and S. A. Buehler (2004), The impact of ozone lines on AMSU-B radiances, Geophys. Res. Lett., 31, L21108, doi:10.1029/ 2004GL021214

Liebe, H. J. (1989), MPM: An atmospheric millimeter-wave propagation model, Int. J. Infrared Millimeter Waves, 10(6), 631-650.

Liebe, H. J., P. W. Rosenkranz, and G. A. Hufford (1992), Atmospheric 60-GHz oxygen spectrum: New laboratory measurements and line parameters, J. Quant. Spectrosc. Radiat. Transfer, 48(5/6), 629-643.

Liebe, H. J., G. A. Hufford, and M. G. Cotton (1993), Propagation modeling of moist air and suspended water/ice particles at frequencies below $1000 \mathrm{GHz}$, in AGARD 52nd Specialists Meeting of the Electromagnetic Wave Propagation Panel, Palma de Mallorca, Spain, AGARD Conf. Proc., 542, 3.1-3.10.

Melsheimer, C., et al. (2005), Intercomparison of general purpose clear sky atmospheric radiative transfer models for the millimeter/submillimeter spectral range, Radio Sci., RS1007, doi:10.1029/2004RS003110.

Rayer, P. J. (2001), NWP SAF microwave transmittances models for RTTOV, technical report, Eur. Org. for the Exploit. of Meteorol. Satell., Darmstadt, Germany.

Rosenkranz, P. W. (1993), Absorption of microwaves by atmospheric gases, in Atmospheric Remote Sensing by Microwave Radiometry, edited by M. A. Janssen, pp. 37-90, John Wiley, Hoboken, N. J.

Rosenkranz, P. W. (1998), Water vapor microwave continuum absorption: A comparison of measurements and models, Radio Sci., 33(4), 919-928. (Correction, 34, 1025, 1999.)

Saunders, R. (2002), RTTOV-7 users guide, report, Met Off., Exeter, U. K. (Available at http://www.metoffice.gov.uk/research/interproj/nwpsaf/rtm/ rttov7_svr.pdf)

Saunders, R., T. Hewison, S. Stringer, and N. Atkinson (1995), The radiometric characterization of AMSU-B, IEEE Trans. Microwave Theory, 43(4), 760-771.

Saunders, R., M. Matricardi, and P. Brunel (1999), An improved fast radiative transfer model for assimilation of satellite radiance observations, Q. J. R. Meteorol. Soc., 125, 1407-1425.

Uppala, S. M., et al. (2006), The ERA-40 reanalysis, Q. J. R. Meteorol. Soc., 131, 2961-3012, doi:10.1256/qj.04.176.

S. A. Buehler, Department of Space Science, Lulea Technical University, Box 812, SE-98128 Kiruna, Sweden. (sbuehler@iru.se)

N. Courcoux, Institute of Environmental Physics, University of Bremen, Otto-Hahn-Allee 1, D-28259 Bremen, Germany. (nathalie@sat.physik. uni-bremen.de)

V. O. John, MPO/RSMAS, University of Miami, 4600 Rickenbacker Causeway, Miami, FL 33149, USA. (vojohn@rsmas.miami.edu) 